\title{
La tricotomía preocupante en la nutrición clínica y su aplicación en los derechos humanos
}

\author{
The troubling trichotomy in clinical nutrition and its application \\ to human rights
}

A preocupante tricotomia na nutrição clínica e sua aplicação aos direitos humanos

\author{
Albert Barrocas ${ }^{1 *}$, Diana Cardenas ${ }^{2}$ \\ Recibido: 5 de junio de 2021. Aceptado para publicación: 3 de agosto de 2021 . \\ https://doi.org/10.35454/rncm.v4supl1.320
}

\section{Resumen}

Introducción: el soporte nutricional (terapia nutricional) es un gran avance de la medicina, que ha permitido alimentar a todos los enfermos que no pueden alimentarse por vía oral. Durante el desarrollo de tecnologías como esta, la falta de previsión de las cuestiones éticas y jurídicas puede resultar problemática. El autor principal de este artículo ha bautizado este enigma como la «tricotomía problemática» (T3).

Objetivo: el objetivo de este artículo teóricamente fundamentado es ofrecer una visión general de los tres componentes de la T3 y su relación con los derechos humanos, así como realizar un análisis de los retos éticos y jurídicos, que impone la innovación en el ámbito de la nutrición clínica a través de las opiniones de algunos líderes.

Métodos: el análisis de los tres componentes de la T3 se basó en la literatura científica y en numerosas fuentes de datos, para ilustrar la relevancia de su interacción, cuando se aplica al campo de la nutrición clínica. Se dirigió una encuesta por correo electrónico a líderes de la nutrición clínica, en la que se les preguntaba por los retos éticos o legales de los futuros avances tecnológicos.

Resultados: los retos generados por los avances en el soporte nutricional deben abordarse desde el enfoque T3, que considera que los aspectos éticos y legales no pueden separarse de los avances tecnológicos. El enfoque ético en el campo de la nutrición clínica puede considerarse

\section{Abstract}

Introduction: Nutritional support is a major advance in medicine which has made it possible to feed all sick people unable to be orally fed. Failure to anticipate ethical and legal issues as part of the development of technologies such as this can be problematic. This conundrum has been dubbed "the troubling trichotomy» (T3) by the lead author.

Objective: The aim of this evidencebased theoretically informed essay is to provide an overview of the three T3 components and their relation to human rights, and to analyze the ethical and legal challenges imposed by innovation in the field of clinical nutrition in the light of selected leading opinions.

Methods: The analysis of the three T3 components was based on academic literature and numerous data sources to illustrate the relevance of their interaction when applied to the field of clinical nutrition. An e-mail survey asking about the ethical or legal challenges of future technological developments was addressed to clinical nutrition leaders.

Results: Challenges created by advances in nutritional support must be addressed from the T3 approach, according to which ethical and legal considerations cannot be separated from technological advances. In the field of clinical nutrition, the ethical approach can be considered as a systematic normative reflection based on a framework of interdependent principles (i.e., principlism), moral values (i.e., attentiveness, responsibility) and actions

\section{Resumo}

Introdução: $\mathrm{O}$ suporte nutricional é um grande avanço na medicina que tem permitido alimentar todos os pacientes que não podem ser alimentados por via oral. Durante o desenvolvimento de tecnologias como esta, a falta de previsão das questões éticas e legais pode ser problemática. $\mathrm{O}$ autor principal (T3) batizou esse enigma de "Tricotomia Problemática".

Objetivo: O objetivo deste ensaio teórico é fornecer uma visão geral dos três componentes da T3 e sua relação com os direitos humanos, bem como uma análise dos desafios éticos e legais que implica a inovação no campo da nutrição clínica através das opiniões de líderes selecionados.

Métodos: A análise dos três componentes da T3 foi baseada na literatura acadêmica e em inúmeras fontes de dados para ilustrar a relevância de sua interação quando aplicada ao campo da nutrição clínica. Uma pesquisa por e-mail foi conduzida com líderes de nutrição clínica perguntando sobre os desafios éticos ou legais de futuros desenvolvimentos tecnológicos.

Resultados: Os desafios gerados pelos avanços no suporte nutricional devem ser enfrentados a partir da abordagem T3, que considera que os aspectos éticos e legais não podem ser separados dos avanços tecnológicos. A abordagem ética no campo da nutrição clínica pode ser considerada como uma reflexão normativa sistemática baseada em um quadro de princípios interdependentes (principia- 
como una reflexión normativa sistemática, basada en un marco de principios interdependientes (principialismo), valores morales (atención, responsabilidad) y acciones que pueden guiar a los profesionales de la salud y a los investigadores ante el futuro desarrollo de las tecnologías. Los aspectos jurídicos deberían tener en cuenta las diferencias nacionales, para enmarcar los retos éticos y la práctica de la disciplina.

Conclusión: la T3 debe considerarse como un enfoque integral, que puede estar en el centro de la nutrición clínica. Con la esperanzadora aceptación mundial del cuidado nutricional como derecho humano, la T3 debería ser menos problemática.

Palabras clave: bioética, principialismo, tecnología, apoyo nutricional. that can guide healthcare professionals and researchers in the face of future technology developments. The legal aspects should consider national differences when framing the ethical challenges and the practice of the discipline.

Conclusion: The troubling trichotomy should be considered as a comprehensive approach that may be at the core of clinical nutrition. As the hope for nutritional care being globally embraced as a human right becomes a reality, the T3 should be less problematic.

Keywords: Bioethics; Principlism; Technology; Nutrition Support. lismo), valores morais (atenção, responsabilidade) e ações que podem orientar aos profissionais de saúde e aos pesquisadores diante o futuro desenvolvimento de tecnologias. Os aspectos legais devem levar em consideração as diferenças nacionais para enquadrar os desafios éticos e a prática da disciplina.

Conclusão: A tricotomia problemática deve ser considerada como uma abordagem integral que pode estar no centro da nutrição clínica. Com a esperançosa aceitação global do cuidado nutricional como um direito humano, a T3 deveria ser menos problemática.

Palavras-chave: Bioética, principialismo, tecnologia, suporte nutricional.
Tulane School of Medicine New Orleans. Luisiana, Estados Unidos.

2 Facultad de Medicina, Universidad El Bosque. Bogotá, Colombia.
*Correspondencia: Albert Barrocas. abarroc@tulane.edu

\section{INTRODUCCIÓN}

Durante muchos años, los profesionales de la salud y otras partes interesadas se enfrentaron a situaciones en las que la tecnología (lo que se puede hacer) suscitaba consideraciones éticas (lo que se debería hacer) que, en última instancia, debían aplicarse en el contexto de la ley (lo que se debe hacer) ${ }^{(1)}$. Barrocas ha bautizado este enigma como la «tricotomía problemática» (T3)

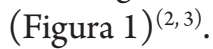

En este artículo se revisarán los tres componentes de la T3 y se centrará en su aplicación en la nutrición clínica, concretamente en el soporte nutricional (también llamado terapia nutricional) y su relación con los derechos humanos.

La nutrición clínica es una ciencia básica interdisciplinaria y aplicada, que se ocupa de la atención de las personas que necesitan cuidados nutricionales (pacientes con desnutrición, ingesta de dietas inadecuadas, carencias de micronutrientes, sobrepeso y obesidad). $\mathrm{Su}$ objetivo es aplicar los principios del cuidado nutricional, para garantizar un equilibrio en el estado de la nutrición, y modular otras funciones biológicas, para influir positivamente en la homeostasis del individuo, en cualquier tratamiento médico y en los resultados de $\operatorname{salud}^{(4,5)}$. El ejercicio de la nutrición clínica se basa en

la aplicación del soporte o terapia nutricional a través del uso de nutrientes por vía oral, enteral o parenteral.

El aspecto «preocupante» de la T3 se basa en su presentación a posteriori. Durante el desarrollo de la tecnología se presta poca o ninguna atención al impacto que los nuevos descubrimientos tienen en los aspectos éticos y legales de su aplicación. La falta de previsión de estos dos componentes de la tricotomía suele ser problemática o preocupante. Algunos desarrollos médicos a lo largo de los años que demuestran este concepto son:

- La definición de la muerte no se cuestionó hasta antes del desarrollo de los respiradores, los medicamentos cardíacos y de reanimación, entre otros.

- El «racionamiento» de la hemodiálisis no se cuestionó hasta el desarrollo de las máquinas de hemodiálisis.

- La selección de personas para recibir trasplantes de órganos no se cuestionó hasta antes del desarrollo de las técnicas quirúrgicas/inmunológicas.

- Los estados vegetativos no se cuestionaron hasta antes del desarrollo de las técnicas, que permiten la supervivencia de los pacientes con lesiones neurológicas graves.

- La mortalidad por desnutrición en individuos incapaces de ingerir alimentos por vía oral no se cues- 


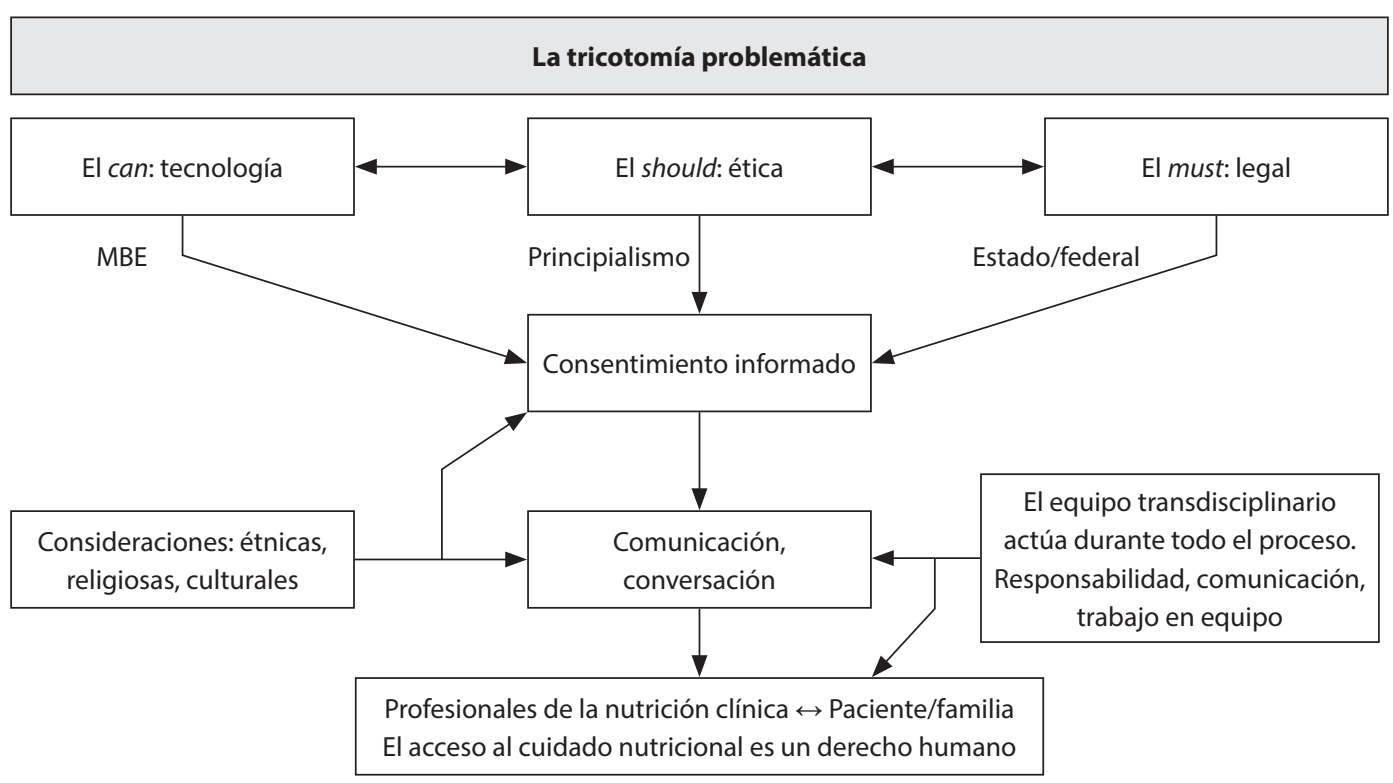

Figura 1. La tricotomía problemática. “Can": lo que se puede hacer ;"should": lo que se debería hacer; "must” : lo que se dede hacer. MBE: medicina basada en evidencia. Realizada por A. Barrocas.

tionó hasta el desarrollo de la nutrición parenteral (NP) total y la nutrición enteral (NE).

La falta de anticipación es problemática, ya que los desarrollos tecnológicos se realizan sin cuestionar los límites necesarios para asegurar un uso responsable de la tecnología y el respeto de principios fundamentales, como la autonomía o el respeto de la dignidad humana. En el caso concreto de la nutrición clínica, la falta de previsión de las cuestiones éticas y jurídicas, y la ausencia de un enfoque ético de la aplicación de la tecnología de la nutrición e hidratación administrada artificialmente (NHA) ignora, entre otros, el principio de proporcionalidad en los cuidados nutricionales, el cual establece que las respuestas deben ser proporcionales al bien que se puede conseguir y al daño que se puede causar $^{(6)}$. Este principio moral adquiere relevancia en el contexto de las cuestiones éticas, relacionadas con la asignación de recursos durante situaciones críticas de escasez, como las pandemias. Además, en lo que respecta a la ética médica, significa que las intervenciones y los riesgos médicos deben ser proporcionales a las posibles vidas salvadas ${ }^{(7)}$, por ejemplo, el análisis de los beneficios frente a las cargas/los riesgos.

\section{TECNOLOGÍA}

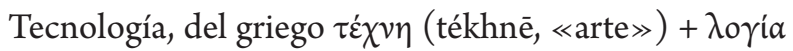
(logía, «estudio»), se refiere a la «ciencia del ofi- cio» ${ }^{(8)}$. La primera idea del concepto de tecnología se encuentra en la antigua Grecia, donde filósofos, como Aristóteles, argumentaban una distinción ontológica fundamental entre $\ll$ cosas naturales $\gg \mathrm{y} \ll$ artefactos ${ }^{(9)}$. Desde entonces, la diferencia fundamental entre los productos fabricados por el hombre y las sustancias naturales ha influido en la reflexión filosófica de la tecnología hasta nuestros días, incluido el enfoque ético. Según el diccionario de Cambridge, la tecnología se define actualmente como el estudio y el conocimiento del uso práctico, especialmente industrial, de los descubrimientos científicos ${ }^{(10)}$.

En el desarrollo de la tecnología suelen ser necesarias consideraciones legales y éticas; entre ellas están las cuestiones del consentimiento informado, el tratamiento ético de las personas y los animales, el respeto y la dignidad, la veracidad de la investigación y los resultados, el análisis de los beneficios frente a la carga/ riesgo, entre otros. Un triste ejemplo en la historia de Estados Unidos sobre los resultados de las experiencias no informadas fue el estudio Tuskegee sobre la sífilis no tratada, también conocido como experimento Tuskegee sobre la sífilis ${ }^{(11,12)}$.

El estudio se realizó entre 1932 y 1972 por el United States Public Health Services. Su objetivo era observar la historia natural de la sífilis no tratada. A los sujetos se les dijo que recibirían asistencia sanitaria gratuita del gobierno federal, pero no recibieron la atención pro- 
metida. Nunca se les informó de su diagnóstico o de los métodos ineficaces, así como de los procedimientos de diagnóstico y los placebos disfrazados de tratamiento. En 1947, la penicilina, que estaba ampliamente disponible, se convirtió en el tratamiento estándar para la sífilis. Ninguno de los hombres infectados fue tratado con el antibiótico. Tras la finalización del proyecto «éticamente abusivo», después de una filtración a la prensa en 1972, se desarrollaron reglamentos y directrices, que incluyen el informe Belmont en $1979^{(13)}$ (debido a la Ley Nacional de Investigación de 1974), el establecimiento de la Oficina para la Protección de la Investigación en Humanos y las Juntas de Revisión Institucional ${ }^{(14)}$.

A nivel internacional, el impulso para el comportamiento ético/legal en la realización de experimentos e investigaciones fueron las conclusiones de las atrocidades del Holocausto, en relación con los abusos médicos nazis. El código de Nuremberg se estableció para proteger los derechos de los sujetos de la investigación ${ }^{(15)}$.

Tabla 1. Respuestas a la encuesta de líderes de opinión en nutrición clínica

\begin{tabular}{|c|c|c|}
\hline Experto & $\begin{array}{c}\text { Previsión de tecnología o avance en la investigación en el campo del } \\
\text { soporte nutricional }\end{array}$ & Consideraciones éticas o legales \\
\hline $\begin{array}{l}\text { Bruce R } \\
\text { Bistrian, MD, } \\
\text { PhD; Boston } \\
\text { MA, EE. UU. }\end{array}$ & 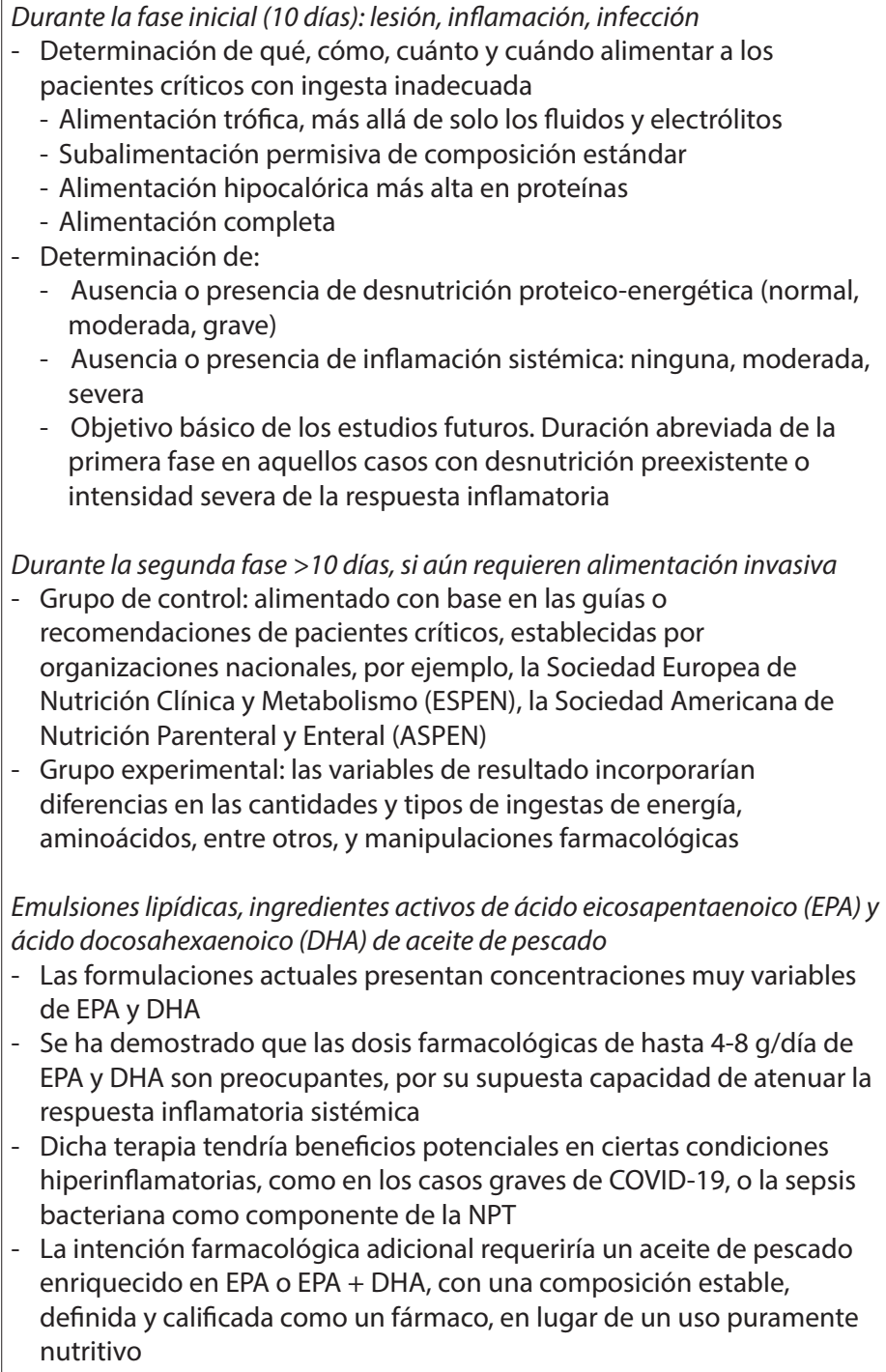 & $\begin{array}{l}\text { - Legalmente, proceso formal de } \\
\text { aprobación de medicamentos } \\
\text { - Se ha estudiado como terapia } \\
\text { independiente, o cuando se combina } \\
\text { con la NPT } \\
\text { - La eficacia puede verse influenciada } \\
\text { por el estado nutricional subyacente }\end{array}$ \\
\hline
\end{tabular}


Tabla 1. Respuestas a la encuesta de líderes de opinión en nutrición clínica (continuación)

\begin{tabular}{|c|c|c|}
\hline Experto & $\begin{array}{c}\text { Previsión de tecnología o avance en la investigación en el campo del } \\
\text { soporte nutricional }\end{array}$ & Consideraciones éticas o legales \\
\hline $\begin{array}{l}\text { Kathy Gura, } \\
\text { PharmD, } \\
\text { BCNSP, FASHP, } \\
\text { FPPA, FASPEN, } \\
\text { FMSHP; } \\
\text { Boston MA, } \\
\text { EE. UU. }\end{array}$ & 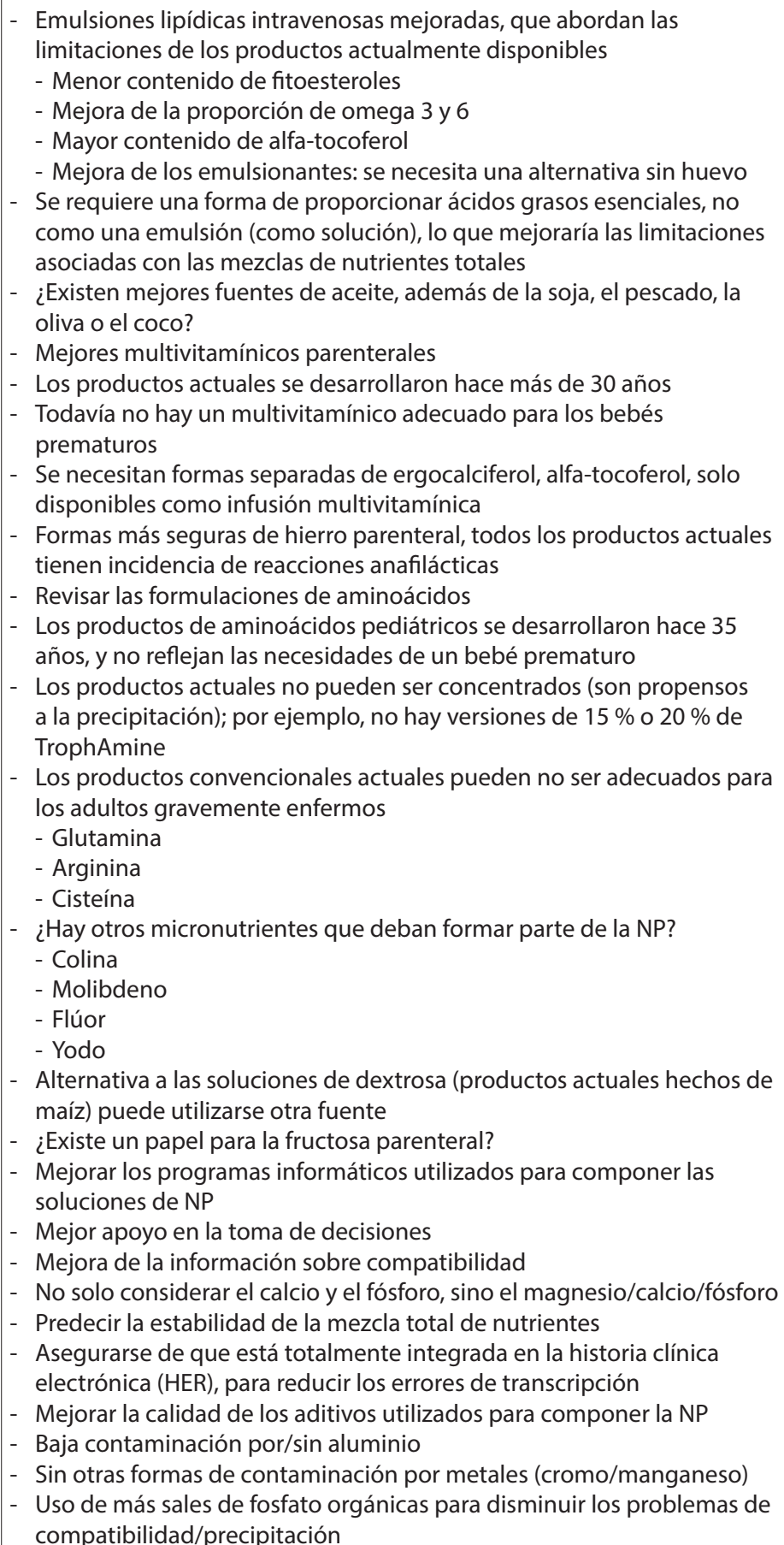 & 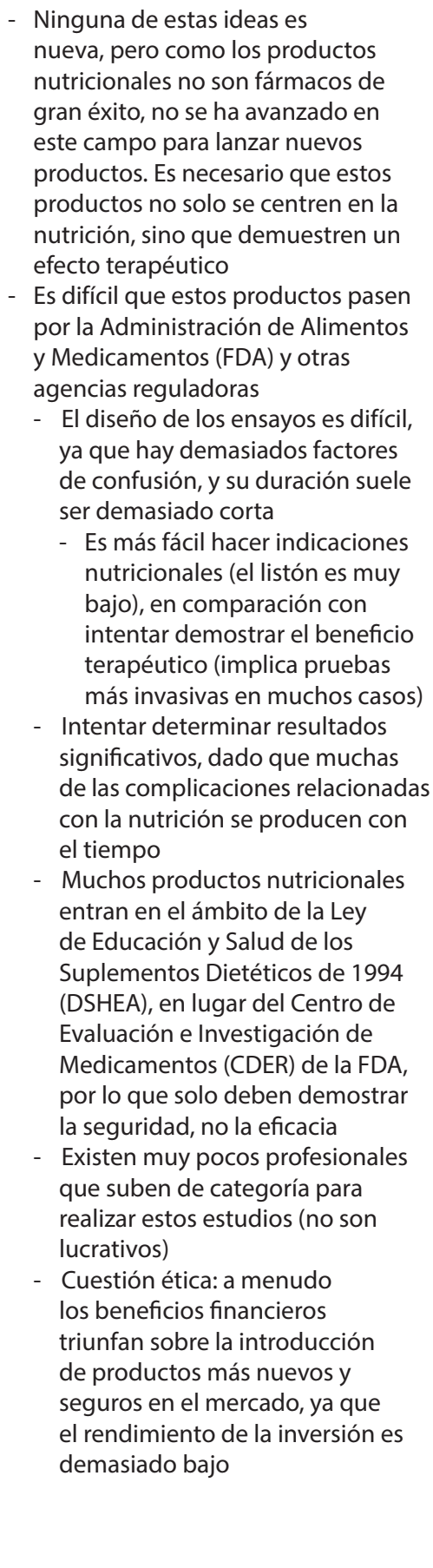 \\
\hline
\end{tabular}


Tabla 1. Respuestas a la encuesta de líderes de opinión en nutrición clínica (continuación)

\begin{tabular}{|c|c|c|}
\hline Experto & $\begin{array}{c}\text { Previsión de tecnología o avance en la investigación en el campo del } \\
\text { soporte nutricional }\end{array}$ & Consideraciones éticas o legales \\
\hline $\begin{array}{l}\text { Dan } \\
\text { Waitzberg, } \\
\text { MD, PhD; Sao } \\
\text { Paolo, Brasil }\end{array}$ & $\begin{array}{l}\text { - Aplicaciones amigables de evaluación nutricional, indicaciones y } \\
\text { prescripciones de terapia nutricional, disponibles independientemente } \\
\text { del patrocinio de la industria } \\
\text { - Inteligencia artificial aplicada a la determinación de qué pacientes } \\
\text { se beneficiarían: tiempo, calorías y proteínas, resultados (morbilidad, } \\
\text { mortalidad, duración de la estancia (LOS), UCI/hospital) } \\
\text { - Seguimiento y evaluación de la terapia nutricional, a través de grandes } \\
\text { bancos de datos }\end{array}$ & $\begin{array}{l}\text { - Anonimato } \\
\text { - Acuerdo entre diferentes hospitales } \\
\text { en el desarrollo de un repositorio } \\
\text { central de datos para procesar la } \\
\text { inteligencia artificial }\end{array}$ \\
\hline $\begin{array}{l}\text { Gil Hardy, } \\
\text { PhD, FRSC, } \\
\text { FASPEN; } \\
\text { Auckland, } \\
\text { Nueva } \\
\text { Zelanda }\end{array}$ & $\begin{array}{l}\text { - Dispositivo de mano (¿aplicación de teléfono móvil?) que utilice la } \\
\text { resonancia magnética, el láser o una tecnología aún por inventar, } \\
\text { que produciría una evaluación inmediata del estado nutricional del } \\
\text { paciente } \\
\text { - Una aplicación complementaria a la anterior, que produzca } \\
\text { automáticamente opciones, con una puntuación para la terapia } \\
\text { nutricional probablemente más práctica y eficaz, para que el clínico } \\
\text { que lo atiende pueda tomar la mejor decisión basada en la evidencia } \\
\text { - Una tecnología mejorada para los pacientes ambulatorios de NP y NE, } \\
\text { utilizando: } \\
\text { - Estabilidad a largo plazo de mezclas de NP verdaderamente «todo } \\
\text { en uno», que contengan todos los micronutrientes y otros nutrientes } \\
\text { actualmente inestables } \\
\text { - Minibombas ambulatorias de alta tecnología para la NP, como las } \\
\text { bombas de insulina utilizadas por los diabéticos tipo } 1 \\
\text { - Formas revolucionarias de administrar los nutrientes, por ejemplo, } \\
\text { utilizar nanotecnología, parches cutáneos, entre otros, para evitar la } \\
\text { contaminación al conectar las sondas de alimentación de la NE, los } \\
\text { catéteres de la NP, los equipos de administración y al paciente } \\
\text { - Nueva tecnología mejorada para reinfusión de quimo en caso de fallo } \\
\text { intestinal } \\
\text { - Impresión 3D del diseño específico para el paciente de intestinos } \\
\text { artificiales y otros órganos, para corregir la insuficiencia intestinal }\end{array}$ & - Ninguna \\
\hline $\begin{array}{l}\text { Sonia } \\
\text { Echeverri, RN, } \\
\text { MSc, FASPEN; } \\
\text { Bogotá, } \\
\text { Colombia y } \\
\text { Portugal }\end{array}$ & $\begin{array}{l}\text { - Fórmulas enterales y mezclas parenterales individualizadas (con } \\
\text { mucha más especificidad hacia la patología, incluido el estado } \\
\text { nutricional y la composición corporal, el momento metabólico, la } \\
\text { microbiota, entre otros) } \\
\text { - Dispositivos médicos más seguros y fáciles de usar para el paciente } \\
\text { (sondas, tubos de alimentación, equipos, bombas de infusión) }\end{array}$ & $\begin{array}{l}\text { - Estos avances en sí mismo no } \\
\text { deberían presentar desafíos éticos } \\
\text { o legales. Lo que representará un } \\
\text { desafío es cómo y quién puede } \\
\text { acceder a estos avances tecnológicos } \\
\text { (justicia distributiva) } \\
\text { - ¿Todos los individuos que los } \\
\text { requieran tendrán derecho a ellos? } \\
\text { empezando por el derecho a la } \\
\text { terapia nutricional administrada } \\
\text { artificialmente (beneficencia) } \\
\text { - ¿Se utilizarán con el debido criterio } \\
\text { médico y la autonomía del paciente? } \\
\text { (autonomía) } \\
\text { - ¿Prevalece el criterio médico o su } \\
\text { uso está influenciado o manipulado } \\
\text { por la industria farmacéutica? (no } \\
\text { maleficencia) }\end{array}$ \\
\hline
\end{tabular}


Tabla 1. Respuestas a la encuesta de líderes de opinión en nutrición clínica (continuación)

\begin{tabular}{|c|c|c|}
\hline Experto & $\begin{array}{c}\text { Previsión de tecnología o avance en la investigación en el campo del } \\
\text { soporte nutricional }\end{array}$ & Consideraciones éticas o legales \\
\hline $\begin{array}{l}\text { Denise Baird } \\
\text { Schwartz, } \\
\text { MS, RD, } \\
\text { FADA, FAND, } \\
\text { FASPEN; } \\
\text { Studio City, } \\
\text { CA, EE. UU. }\end{array}$ & $\begin{array}{l}\text { - Abordar cualquier opción del sustrato (nutriente) y la administración } \\
\text { que mejore la relación beneficio frente al riesgo adverso de la nutrición } \\
\text { e hidratación artificialmente administrada } \\
\text { - Diseñar y publicar estudios de investigación para corroborar que la } \\
\text { calidad de vida del individuo se puede optimizar con estas mejoras en } \\
\text { el equipo y la administración de nutrientes } \\
\text { - Que los clínicos conozcan los resultados de la investigación y utilicen la } \\
\text { información en la discusión con el paciente o el responsable sustituto, } \\
\text { con la toma de decisiones compartida en el ámbito clínico con todos } \\
\text { los pacientes } \\
\text { - La incorporación de los puntos anteriores permitiría abordar los } \\
\text { cuatro principios éticos de respeto a la autonomía, beneficencia, no } \\
\text { maleficencia y justicia }\end{array}$ & $\begin{array}{l}\text { - Los aspectos éticos se abordarían } \\
\text { para cada punto al diseñar el estudio } \\
\text { de investigación } \\
\text { - Los aspectos legales variarían según } \\
\text { los distintos estados, regiones, } \\
\text { países, entre otros }\end{array}$ \\
\hline
\end{tabular}

NE: nutrición enteral; NP: nutrición parental; NPT: nutrición parenteral total; UCl: unidad de cuidados intensivos.

El concepto de consentimiento informado se estableció por la Declaración de Helsinki de la Organización Mundial de la Salud (OMS) en 1964(15). La recientemente adoptada Declaración de Cartagena ${ }^{(16-18)}$ y el documento de posición «Nutrición clínica y derechos humanos. Un documento de posición internacional» son una extensión de este último documento ${ }^{(4)}$.

Mirando hacia el futuro y centrándose en la nutrición clínica, los autores se pusieron en contacto con un grupo de líderes de opinión en el ámbito de la nutrición clínica/el apoyo a la nutrición y solicitaron su aportación en dos áreas:

1. ¿Qué tecnología/investigación preveían en el campo del soporte nutricional?

2. ¿Qué consideraciones éticas o legales suscitaría la aplicación de la investigación?

Los resultados de la encuesta se resumen en la Tabla 1 .

La opinión de los líderes muestra las oportunidades de desarrollo tecnológico en el campo del soporte nutricional, la nutrición clínica y los desafíos éticos y legales. La mayoría de ellos coinciden con la necesidad de desarrollos tecnológicos en cuanto a productos y dispositivos de nutrición. La afirmación de esta necesidad de desarrollo se basa en el hecho de que los productos nutricionales han evolucionado poco en las últimas dos décadas. Los nuevos productos y dispositivos responderían a la necesidad de suministrar nutrientes a pacientes con necesidades particulares, debido a la adaptación metabólica a la enfermedad. Esto implica considerar la respuesta metabólica a la enfermedad como adaptativa, y que el soporte nutricional no es el mero suministro de nutrientes (como puede considerarse la alimentación de una persona sana), sino que debe pensarse como un tratamiento médico. Esto significa que el suministro de nutrientes, calorías y proteínas responde a una respuesta adaptativa a la enfermedad, y no a la falta de nutrientes por simple inanición. Hay que reconocer que esta respuesta adaptativa a veces puede convertirse en «patológica» ${ }^{(19)}$. El desarrollo de los conocimientos científicos sobre el metabolismo debería ayudar a identificar dónde está el límite entre una respuesta normal y una patológica. La metabolómica y la inteligencia artificial (IA) podrían apoyar el desarrollo de estos campos, especialmente en el proceso de toma de decisiones. Los planteamientos éticos y jurídicos serían fundamentales para estos desarrollos ${ }^{(19)}$.

Dos líderes de opinión mencionaron el posible impacto ético de la industria farmacéutica en las decisiones médicas. El desarrollo y la innovación de los productos de terapia nutricional deben llevarse a cabo teniendo en cuenta los principios de justicia, beneficencia y no maleficencia, y las decisiones médicas sobre su uso deben ser independientes de cualquier influencia comercial.

Una de las opiniones considera que no es el desarrollo de productos nutricionales en sí lo que podría tener implicaciones legales o éticas, sino lo que se hace con ellos. Esto puede aplicarse a todos los desarrollos tecnológicos. De hecho, no es el desarrollo en sí mismo, sino lo que los humanos hacen con él. El reto ético sería crear 
productos nutricionales rentables, que sean capaces de proporcionar la mejor relación beneficios/riesgos-perjuicios, de acuerdo con el conocimiento emergente.

La metabolómica y la IA pueden poner en peligro el manejo confidencial de los datos de los pacientes. Los desarrollos de la IA pueden aplicarse mediante el enfoque de los 4 principios, al que habría que añadir un quinto principio, la «explicabilidad», que debe entenderse como la suma de la inteligibilidad (icómo funciona?) y la responsabilidad (¿quién es responsable de su funcionamiento? $)^{(20)}$

\section{ÉTICA}

La ética, el segundo componente de la T3, es fundamental, ya que no solo abarca el comportamiento esperado, sino los valores individuales que pueden entrar en conflicto. El término «ética» deriva de la palabra griega ethos, mientras que el término «moral» proviene de la palabra latina mores, ambas se refieren a «costumbres o comportamientos comunes» ${ }^{(21)}$. Debido a que originalmente tenían el mismo significado, con frecuencia ambos términos se utilizan indistintamente, aunque no signifiquen lo mismo. La moral se refiere a un conjunto de normas y principios comunes a un grupo social. Esta se encuentra profundamente influenciada por varios factores culturales, como la religión, la historia, la tradición, la educación, las creencias, entre otros. La ética es una rama de la filosofía, que pretende realizar un análisis intelectual de la dimensión humana moral en toda su complejidad. La ética se ocupa de los principios que nos permiten tomar decisiones sobre lo que está bien y lo que está mal. En otras palabras, la ética es el estudio de lo que es moralmente correcto y lo que no. Se refiere a un juicio sobre los comportamientos, buenos o malos.

Cuando se aplica a las ciencias biomédicas, la ética se enfrenta a una ambigüedad, ya que abarca indistintamente la ética médica y la bioética, a la que se puede añadir la tecnoética (Tabla 2).

La ética médica, la bioética y la tecnoética, concebidas como subcampos de la ética, tienen ámbitos de aplicación cercanos, pero también comparten algunos principios y enfoques, que se pueden aplicar en el análisis de ciertas disciplinas, como para la nutrición clínica.

Cuando se trata de analizar la práctica de la nutrición clínica a través del enfoque ético hay que desta-

Tabla 2. Subcampos de la ética

\begin{tabular}{|c|c|c|}
\hline Subcampos & Definición & Enfoques y teorías morales \\
\hline Ética médica & $\begin{array}{l}\text { Se ocupa de las cuestiones éticas relacionadas con la práctica médica. Desde } \\
\text { la antigüedad, la profesión médica ha suscrito un conjunto de principios y } \\
\text { declaraciones éticas, desarrolladas principalmente en beneficio del paciente. } \\
\text { Estos principios, integrados en los códigos deontológicos, no son leyes, sino } \\
\text { normas de conducta, que definen lo esencial del comportamiento honorable del } \\
\text { médico }\end{array}$ & \multirow{2}{*}{$\begin{array}{l}\text { - Enfoque principialista: } \\
\text { autonomía, beneficencia, no } \\
\text { maleficencia, justicia } \\
\text { - Enfoque basado en los } \\
\text { derechos humanos } \\
\text { - Teorías de la justicia } \\
\text { distributiva (utilitarismo) } \\
\text { - Teoría moral deontológica } \\
\text { - Ética de la virtud }\end{array}$} \\
\hline Bioética & $\begin{array}{l}\text { Se ocupa específicamente de las cuestiones éticas, que han surgido en el } \\
\text { ámbito de los organismos vivos con el progreso médico y científico. El término } \\
\text { «bioética», procedente de las palabras griegas bios y ethos, se utilizó por primera } \\
\text { vez en } 1927, \text { por el teólogo protestante Fritz Jahr; pero no fue hasta } 1970 \text { cuando } \\
\text { el término se desarrolló por Van Rensselaer Potter en el libro Bioethics, a bridge } \\
\text { to the future }{ }^{(23)} \text {. Llamó la atención sobre el hecho de que los rápidos avances de } \\
\text { la ciencia se habían producido sin prestar la debida atención a los valores. Potter } \\
\text { concibió esta nueva disciplina como un «puente» entre los hechos y los valores }\end{array}$ & \\
\hline Tecnoética & $\begin{array}{l}\text { También conocida como ética de la tecnología. Se considera como un área de } \\
\text { investigación interdisciplinaria, que se basa en teorías y métodos de múltiples } \\
\text { dominios del conocimiento (como las comunicaciones, los estudios de ciencias } \\
\text { sociales de la información, los estudios de la tecnología, la ética aplicada y la } \\
\text { filosofía) para proporcionar ideas sobre las dimensiones éticas de los sistemas } \\
\text { tecnológicos y las prácticas para el avance de una sociedad tecnológica }{ }^{(24)} \text {. Se } \\
\text { centra en la reflexión sobre los usos éticos de la tecnología y la proteción contra } \\
\text { el mal uso de la misma }{ }^{(24,25)}\end{array}$ & $\begin{array}{l}\text { - Enfoques culturales y } \\
\text { políticos } \\
\text { - } \text { La responsabilidad } \\
\text { - } \text { Según Hans Jonas, la } \\
\text { tecnología requiere una ética } \\
\text { en la que la responsabilidad } \\
\text { es el imperativo central } \\
\text { porque «por primera vez en } \\
\text { la historia podemos destruir } \\
\text { la Tierra y la humanidad»(26) }\end{array}$ \\
\hline
\end{tabular}


car que la nutrición es capaz de presentarse como una forma legítima de cuidado en la práctica médica ${ }^{(19)}$. La alimentación de los pacientes se convierte en una forma de cuidado, pero también de tratamiento, lo que plantea nuevas cuestiones éticas cuando se aplica a situaciones concretas. La cuestión relevante aquí es saber qué características éticas son fundamentales para el desarrollo tecnológico del soporte nutricional, y su aplicación en la práctica clínica.

\section{MARCO ÉTICO EN EL ÁMBITO DE LOS CUIDADOS NUTRICIONALES}

\section{El principio de respeto a la vulnerabilidad humana y a la integridad personal del paciente desnutrido}

El principio de respeto a la vulnerabilidad humana expresa la preocupación por la fragilidad del ser humano. La palabra vulnerable procede del latín vulnerabilis, formada por vulnus (herida) y el sufijo abilis (capaz, que indica posibilidad); por tanto, una persona vulnerable es un individuo que puede ser fácilmente herido y no puede defenderse fácilmente. La vulnerabilidad es una dimensión ineludible de la vida de las personas y de la configuración de las relaciones humanas ${ }^{(27)}$. De este modo, la ley considera que las personas vulnerables deben ser protegidas para que no sean objeto inadecuado de investigación o de cualquier coacción. La cuestión del respeto y el acompañamiento de la persona vulnerable va más allá de la protección de una categoría de individuos y del problema del consentimiento informado. Esta cuestión no se refiere exclusivamente a la dimensión jurídica, ni siquiera esencialmente, sino a una dimensión ética.

En el campo de la ética, la noción de la vulnerabilidad no es solo una descripción neutra de la condición humana, sino una prescripción normativa, que implica ocuparse de la vulnerabilidad. En el ámbito de la nutrición clínica se considera que el paciente desnutrido o con riesgo de desnutrición tiene una «vulnerabilidad especial», esto significa que la persona es frágil y necesita a otras personas para satisfacer sus necesidades materiales básicas ${ }^{(19,27)}$.

Un paciente desnutrido o con riesgo de desnutrición es vulnerable porque su integridad física y psicológica está comprometida, así como su calidad de vida. La persona es frágil por su situación de dependencia. Esto se explica porque se encuentra debilitada y necesita los conocimientos de los demás, especialmente de los profesionales sanitarios, para mejorar. Esta vulnerabilidad se ve agravada por el hecho de que la malnutrición o las enfermedades y afecciones relacionadas con la malnutrición carecen de reconocimiento y sensibilización por parte de los profesionales de la salud de forma más general. Esto puede explicarse, en parte, por la falta de educación nutricional, tanto a nivel de grado como de posgrado ${ }^{(28,29)}$. Así, la desnutrición implica una «doble vulnerabilidad» para el paciente: la primera, la del detrimento físico o mental; mientras que la segunda se da por el alto desconocimiento y la falta de educación sobre la nutrición clínica entre los profesionales sanitarios ${ }^{(19)}$.

El reconocimiento de esta particular vulnerabilidad del paciente desnutrido conlleva el reconocimiento de la responsabilidad del profesional de salud, y sirve para la elaboración de políticas públicas sobre la necesidad de poner más recursos a su disposición. En este sentido, el compromiso con el respeto a la vulnerabilidad humana y a la integridad personal es un componente obligatorio de las responsabilidades de políticos, legisladores, reguladores, administradores y profesionales sanitarios.

\section{Responsabilidad del profesional de la salud ante el paciente desnutrido}

El sentido ético más profundo de la vulnerabilidad implica un compromiso de responsabilidad hacia los demás ${ }^{(30)}$. La responsabilidad no debe concebirse, en su estrecha dimensión jurídica, como una obligación de cuidar a los demás y, en consecuencia, de ser objeto de sanciones si no se garantiza ese cuidado. Por el contrario, según Emanuel Levinas, la relación ética comienza con la respuesta al otro. Así, la relación con el otro es el punto de partida de la ética. Ser responsable del otro es esencialmente ser una «sustitución» de este ${ }^{(31)}$. Según este filósofo francés, esto significa ponerme en el lugar del otro, no subordinarlo según mis deseos, sino ofrecerle lo que necesita, empezando por las necesidades materiales básicas. Además, la sustitución significa que los profesionales de la salud deben transmitir la voluntad del paciente ${ }^{(31)}$.

En el caso de los cuidados nutricionales, la desnutrición puede llevar a la necesidad de soporte nutricional, y esta debe considerarse como una intervención médica, que requiere una indicación para alcanzar un objetivo de tratamiento y del consentimiento informado del paciente ${ }^{(32)}$. Esto significa, en primer lugar, que además de considerar al paciente por su capacidad de autonomía (para tomar decisiones sobre la terapia nutricional) es necesario tener en cuenta su doble vulnerabilidad; en segundo lugar, esto indica que no debe ser el miedo al 
castigo lo que guíe a los profesionales en su práctica del cuidado nutricional, sino la responsabilidad ética de tratar y dar las necesidades nutricionales básicas ${ }^{(19)}$.

La responsabilidad de proporcionar las necesidades nutricionales individuales y específicas de cada paciente recae en los profesionales de la salud. El reto para estos últimos es, en primer lugar, garantizar una evaluación y un diagnóstico adecuados de la desnutrición y, a continuación, asegurar este tratamiento y satisfacer esta necesidad vital. El conflicto puede surgir cuando los pacientes o las familias no están de acuerdo con la retención o la retirada de la terapia nutricional en algunas situaciones particulares. La terapia nutricional médica se encuentra en constante tensión entre el «cuidado» (care) y la «cura» (cure). El origen del problema está en la forma en que se concibe la terapia nutricional médica: como parte de un tratamiento médico o como un cuidado (comida y agua). Para comprender mejor esta dualidad es necesario entender qué es un «nutriente artificial», más allá de su dimensión bioquímica, y cómo el desarrollo tecnológico de la nutrición artificial ha permitido alimentar a cualquier paciente en la actualidad ${ }^{(5)}$.

La cuestión relevante aquí es saber cómo deben actuar los profesionales de salud a la hora de alimentar al paciente enfermo. ¿Cuál es la mejor manera de atender a un paciente concreto en un momento determinado? La teoría de la ética del cuidado (ethics of care) de Joan Tronto ${ }^{(33)}$ es un enfoque que puede ayudar a responder estas preguntas.

\section{LA ÉTICA DEL CUIDADO}

\section{El proceso de cuidado nutricional y las fases del cuidad}

La ética del cuidado es una teoría normativa, que sitúa el fenómeno del cuidado en el centro de la reflexión ética. Se desarrolla a partir de la comprensión del ser humano como un ser relacional vulnerable e interdependiente. Mientras que las teorías morales tradicionales, es decir, el utilitarismo, la deontología, la teoría de la justicia y la ética de la virtud, se basan en la primacía de la autonomía, la ética del cuidado, más contextualizada y concreta, hace hincapié en la noción de vulnerabilidad, que considera una de las características esenciales de la condición humana. La ética del cuidado destaca la importancia de la respuesta, por lo que la pregunta ética es ¿Cómo responder? ${ }^{(33)}$.

La ética del cuidado, definida por Tronto como una práctica y una disposición, es un proceso continuo, que consta de 5 fases del cuidado y sus características morales ${ }^{(33)}$. Estos elementos morales son actitudes y habilidades específicas, necesarias para un cuidado eficaz. Esta teoría normativa es útil para definir algunas características éticas, necesarias para un buen cuidado nutricional. Así, las fases del cuidado pueden corresponder a los 7 pasos del proceso de cuidado nutricional, como se propone en la Figura 2.

Según las guías de la ESPEN sobre las definiciones y la terminología de la nutrición clínica, la atención nutricional debe proporcionarse en una secuencia sistemática, que incluye 7 pasos distintos interrelacionados. Esta secuencia sistemática se denomina proceso de atención nutricional ${ }^{(34)}$.

El primer y el segundo paso son el tamizaje del riesgo de desnutrición y la evaluación nutricional, que pueden concebirse como la fase de «preocuparse por» (caring about) de la ética del cuidado. Esta primera fase requiere un elemento ético: el de la atención, es decir, una consideración justa y afectuosa de una realidad individual ${ }^{(33)}$. De forma práctica, esto significa que los profesionales de la salud reconocen la necesidad de cuidado nutricional mediante la identificación de los sujetos desnutridos o en riesgo nutricional, por medio del uso de una herramienta adecuada y validada en todos los sujetos que entran en contacto con los servicios de salud, y al evaluar el estado nutricional del paciente ${ }^{(19)}$. En este sentido, la necesidad de un consenso en la comunidad científica sobre los criterios diagnósticos ${ }^{(35)}$ y la naturaleza de la desnutrición asociada con la enfermedad ${ }^{(36)}$ son fundamentales para asegurar un adecuado cuidado nutricional.

La atención a la desnutrición lleva a establecer una respuesta adecuada, que se consolida en la fase de «encargarse de» (taking care of). En esta fase, los profesionales de la salud reconocen su responsabilidad para responder al riesgo de desnutrición de un paciente, o a cualquier grado de desnutrición, y llegan a un diagnóstico. Esta responsabilidad es compartida y concierne a todos los actores implicados en la atención. El profesional sanitario, mediante la elaboración de un plan nutricional, se asegurará de que existen todas las condiciones necesarias para satisfacer las necesidades nutricionales del paciente. En esta fase, las acciones tienen principalmente dos propósitos específicos: combatir la desnutrición y limitar la pérdida de calidad de vida mediante el soporte nutricional. Cabe destacar que la responsabilidad es una responsabilidad desde la ética y no debe concebirse como una responsabilidad basada en la obligación legal (deber) ${ }^{(19)}$. 


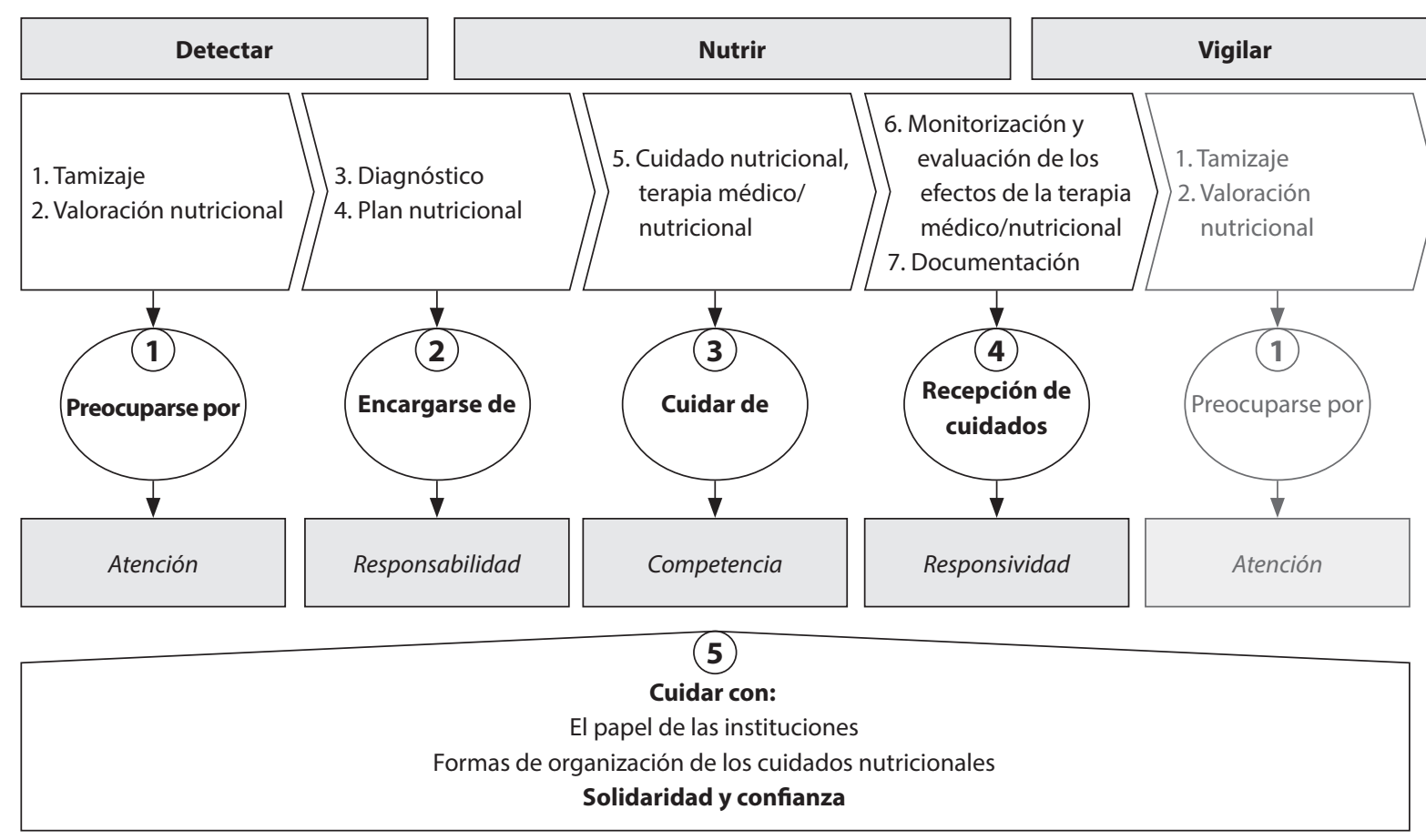

Figura 2. Las 5 fases de la ética del cuidado y sus elementos éticos, integrados en el proceso de cuidado nutricional. Las palabras resaltadas en negrita en los círculos representan las fases de la atención, mientras que las cursivas son los elementos éticos requeridos en cada fase.

La tercera fase de la ética del cuidado es «cuidar de» (care giving), que implica la actividad directa de contacto con el paciente. Los profesionales de la salud deben ser capaces de responder de la mejor manera posible a las necesidades nutricionales del paciente, aportando nutrientes por vía oral, por sonda enteral o por NP, para prevenir o tratar la desnutrición e impactar en los desenlaces clínicos de forma individualizada. En esta fase, la competencia se considera un elemento ético. No se puede simplemente reconocer la necesidad de cuidar, aceptar la responsabilidad y seguir adelante sin la suficiente competencia o habilidad adecuada. Esa falta de competencia haría que no se cubrieran las necesidades de cuidado o atención, o que aumentara el riesgo de complicaciones; por tanto, los profesionales de salud deben comprometerse con el aprendizaje continuo y permanente, para garantizar la competencia en la práctica de los cuidados nutricionales ${ }^{(19)}$.

Por último, en la cuarta fase «recepción de los cuidados» (care receving), los profesionales de la salud evalúan el éxito del soporte nutricional con el paciente y documentan este proceso. Esta fase es esencial para preservar la relación entre el paciente y el profesional de la salud. El elemento ético de esta fase es la capacidad de respuesta, o responsividad (responsiveness), que se refiere a la capacidad de respuesta del receptor de los cuidados; es decir, a la forma en que el paciente o la familia perciben los cuidados $^{(19)}$. Según la ética del cuidado, la realización de un proceso completo de cuidado nutricional por parte del profesional de salud significa que este está respondiendo a la vulnerabilidad del paciente.

La ética de los cuidados destaca dos consideraciones fundamentales: en primer lugar, los cuidados se refieren a un ser que sufre una condición patológica que podemos tratar o prevenir; en segundo lugar, cuando cuidamos a alguien, a un individuo o un ser, esto significa que nos preocupamos por las dimensiones emocional, social y psicológica. En consecuencia, el cuidado no es solo una cuestión de suministrar un tratamiento para aliviar o curar, sino que es una cuestión de humanidad. En ese sentido, la terapia nutricional, como terapia médica, es capaz de curar o prevenir la desnutrición, impactar en los desenlaces clínicos y ayudar en la recuperación de la enfermedad, pero también es un apoyo a la alimentación nutricional básica. 


\section{ASPECTOS LEGALES}

A pesar de lo que se puede y se debe hacer, en última instancia, nuestro comportamiento se rige por lo que se debe o lo que se nos permite hacer según las leyes. La práctica de los profesionales de la salud está regulada por leyes y normas, que se encuentran en diferentes documentos normativos (constitución, leyes, normas, reglas, reglamentos, códigos).

La bioética o ética médica presupone un sistema normativo, que inicialmente no pertenece al derecho, sino a la moral o a la ética ${ }^{(37)}$. En particular, la bioética surge como un enfoque para analizar y reflexionar sobre los retos sociales y éticos, que imponen el desarrollo médico y tecnológico, como es la nutrición artificial. A través de la reflexión bioética pueden evolucionar los pensamientos y las legislaciones. Así, la bioética se considera una forma de influir en las legislaciones relativas a estos avances. La reflexión bioética «tiende a su consagración jurídica» ${ }^{(37)}$. El proceso de generación de la norma se inicia en un marco ético, lo que solo se convierte en norma jurídica mediante su consagración con la adopción de la ley. Esta es la primera fuente jurídica a la que hay que añadir otras fuentes, como las extralegales, es decir, fuentes constitucionales, de derecho internacional y reglamentarias, así como otras específicas de la bioética, como las que emanan de los comités de bioética ${ }^{(35)}$.

El marco jurídico normativo de la práctica de la asistencia nutricional varía de un país a otro; por ejemplo, en Francia, la primera ley de bioética se promulgó en 1994 y ha ido evolucionando hasta la actual de ClaeysLéonetti, en 2016 (actualmente en revisión). El estado de la nutrición artificial ha ido evolucionando a través de la legislación, para finalmente reconocer que alimentar a los pacientes por cualquier medio (NE o NP) es un tratamiento médico. Esto ha llevado a considerar que las cuestiones relativas a la introducción o la retención/retirada (renuncia) de la nutrición artificial siguen el mismo enfoque que para otros tratamientos de soporte vital.

En Latinoamérica, las leyes basadas en la bioética son escasas. Algunos países, como Argentina, Colombia, Uruguay y Chile, cuentan con leyes y regulaciones sobre cuidados paliativos. Por ejemplo, en Colombia, la Ley 1733 de 2014 y la Resolución 1216 de 2015 reconocen el derecho del paciente a rechazar tratamientos extraordinarios, entre ellos, la nutrición artificial.
Por otra parte, según un estudio reciente existe una alta heterogeneidad en la normativa del cuidado nutricional $^{(38)}$. La Tabla 3 muestra las legislaciones y regulaciones nacionales en materia de atención nutricional en 17 países latinoamericanos. En consecuencia, en Latinoamérica existe una importante ambigüedad sobre el estado de los productos nutricionales para la terapia nutricional y una escasez de leyes basadas en la bioética, que podría conducir a dilemas éticos en la práctica del soporte nutricional.

El sistema jurídico estadounidense se resume en la Figura 3. Publicaciones previas ofrecen un análisis más detallado ${ }^{(39,40)}$. Aunque no existen legislaciones específicas que regulen la nutrición clínica en sí, se espera que se preste una atención en salud adecuada. Una atención inadecuada puede dar lugar a litigios penales y, con mayor frecuencia, a civiles. Bajo los auspicios de la negligencia, una persona que no reciba una nutrición adecuada, y que esto provoque un resultado adverso, puede presentar cargos legales basados en la regla $A B C D$, que abarca: aceptación (relación establecida entre el paciente y los profesionales sanitarios); incumplimiento (el deber de proporcionar el estándar de atención); causa (la etiología del evento adverso; y daño (resultante del evento adverso) ${ }^{(40)}$.

En otro orden de ideas, como ocurre en otros países, la nutrición artificial se considera un tratamiento médico, como los respiradores y la hemodiálisis, que puede retenerse o retirarse (renunciar) en determinadas circunstancias, respetando la autonomía y la dignidad del paciente o del responsable sustituto; sin embargo, a raíz del caso Terri Schiavo ${ }^{(41)}$, varios estados establecieron un requisito más rígido en lo que respecta a la nutrición artificial. A menos que se indique específicamente en una directiva anticipada, no se puede renunciar a la nutrición artificial sin pruebas claras y convincentes.

Además de las leyes federales y estatales, los organismos reguladores y las aseguradoras, por ejemplo, la Comisión Conjunta, el Medicare y el Medicaid, exigen intervenciones nutricionales específicas, que incluyan la detección, la evaluación, el diagnóstico y el tratamiento no solo en el hospital, sino en entornos sanitarios alternativos, como el hogar, las residencias de ancianos, los hospitales de cuidados agudos a largo plazo, entre otros. Más que un recurso legal, aquel que infringe estas normas se enfrenta a una posible denegación o reducción del reembolso (Tabla 4). 
Tabla 3. Legislaciones y regulaciones nacionales latinoamericanas en materia de atención nutricional

\begin{tabular}{|c|c|c|c|c|c|c|c|c|c|}
\hline \multirow[t]{2}{*}{ País } & \multicolumn{9}{|c|}{ Categorías de nutrición clínica } \\
\hline & NP & NE & SNO & APME & ETN & DM & DM & NAD & PCN \\
\hline Argentina & Sí & Sí & Sí & Sí & Sí & Sí & Sí & No & No \\
\hline Bolivia & No & No & Sí & No & Sí & No & Sí & No & No \\
\hline Brasil & Sí & Sí & Sí & Sí & Sí & Sí & Sí & No & No \\
\hline Chile & Sí & No & Sí & No & No & No & Sí & Sí & No \\
\hline Colombia & No & No & Sí & Sí & No & Sí & No & No & No \\
\hline Costa Rica & No & No & Sí & No & No & No & Sí & No & No \\
\hline Ecuador & No & No & Sí & No & No & No & Sí & No & No \\
\hline El Salvador & No & No & Sí & No & No & No & Sí & No & No \\
\hline Guatemala & No & No & Sí & Sí & No & No & No & No & No \\
\hline México & Sí & Sí & Sí & No & Sí & Sí & Sí & Sí & No \\
\hline Panamá & No & No & Sí & No & Sí & No & Sí & Sí & No \\
\hline Paraguay & Sí & Sí & Sí & Sí & Sí & Sí & Sí & No & No \\
\hline Perú & Sí & Sí & Sí & No & Sí & No & Sí & No & No \\
\hline República Dominicana & Sí & No & Sí & No & No & No & No & No & No \\
\hline Uruguay & No & No & Sí & No & No & No & Sí & No & No \\
\hline Venezuela & No & No & Sí & No & Sí & Sí & No & Sí & No \\
\hline
\end{tabular}

APME: alimentos para propósitos médicos especiales; CM: central de mezclas; DM: dispositivos médicos; ETN: equipos de terapia nutricional; NAD: nutrición a domicilio; NE: nutrición enteral; NP: nutrición parenteral; PCN: proceso de cuidado nutricional (tamizaje, diagnóstico, terapia nutricional, monitorización); SNO: suplementos nutricionales orales. Modificado de ${ }^{(36)}$.

\section{APLICACIÓN DE LA T3 A LOS DERECHOS HUMANOS}

Bajo el liderazgo y el estímulo de la Federación Latinoamericana de Nutrición Clínica, Terapia Nutricional y Metabolismo (FELANPE) se ha logrado un esfuerzo global para establecer el acceso a la nutrición como un derecho humano ${ }^{(4)}$. Existe tecnología para proporcionar nutrición a todos los individuos, incluidos aquellos que no pueden ingerir ningún nutriente o uno suficiente por la vía oral. Las preocupaciones legales se centrarían en el desarrollo de estatutos, que dictarían cómo lograr el objetivo del acceso universal a la atención nutricional, a pesar de los limitados recursos financieros y otros logísticos.

El cuidado nutricional debe considerarse un derecho humano fundamental, intrínsecamente vinculado al derecho a la alimentación, así como al derecho a la salud. El derecho a la salud incluye el acceso a una atención sanitaria oportuna, aceptable, de calidad y asequible. Este análisis puede contribuir a la construcción de una perspectiva moral, política y jurídica, a nivel mundial, del concepto de cuidado nutricional. Además, puede ser la piedra angular de los fundamentos de los instrumentos políticos y jurídicos en el ámbito de la nutrición clínica.

Reconocer que el cuidado nutricional es un derecho humano no implica que exista la obligación de alimentar a todos los pacientes en cualquier etapa de la vida y a cualquier costo. Por el contrario, este derecho implica, desde un punto de vista ético, que se debe tomar la mejor decisión para el paciente, esto puede incluir, en algunas circunstancias, la decisión de no alimentarlo.

Los derechos humanos y la ética están estrechamente relacionados, ya que se apoyan y complementan 


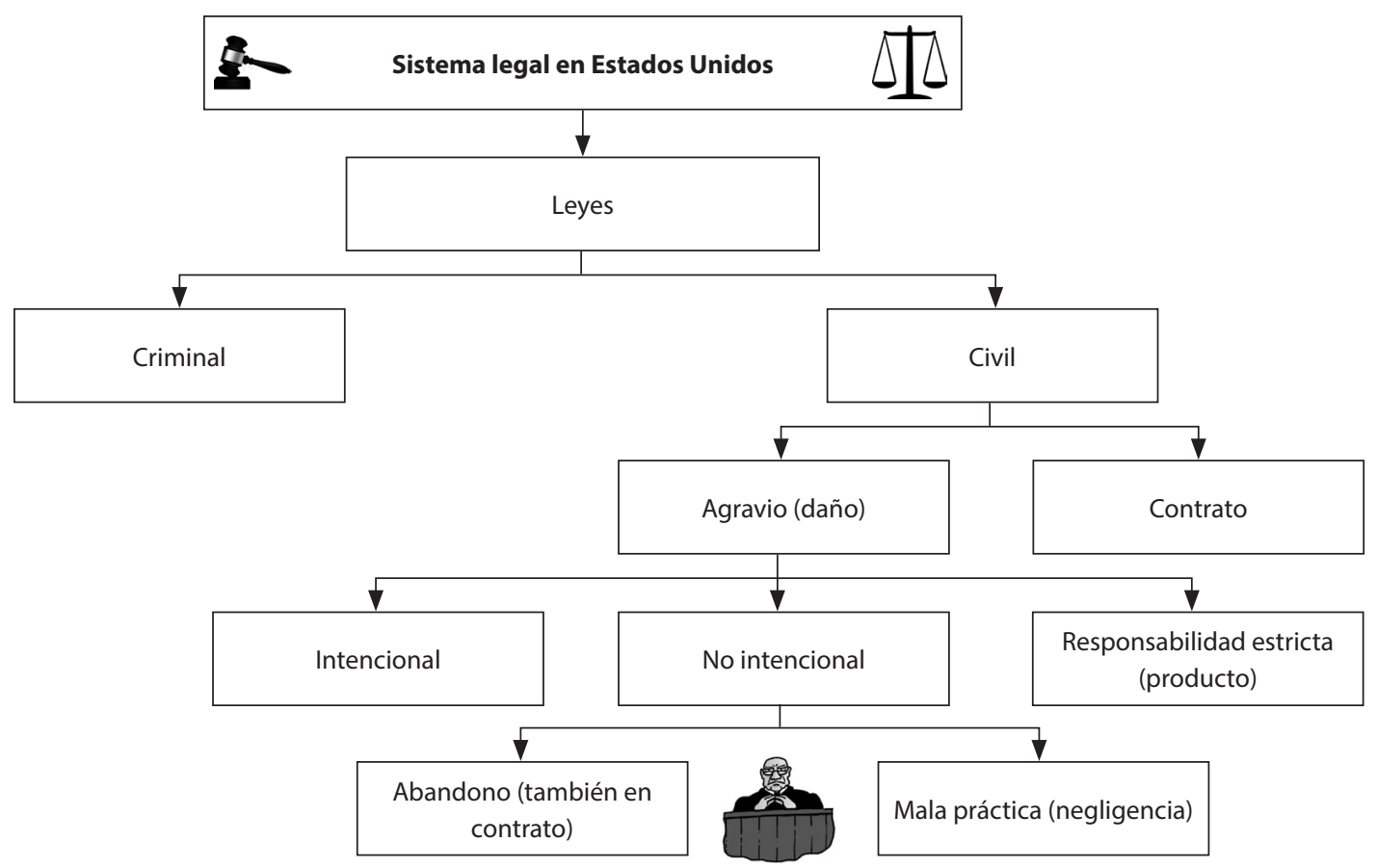

Figura 3. Sistema jurídico estadounidense. Realizado por A. Barrocas.

Tabla 4. Resumen de los fundamentos éticos de la práctica del apoyo nutricional y ejemplos de fuentes legales y deontológicas para el desarrollo tecnológico y la innovación en la nutrición clínica

\begin{tabular}{|c|c|c|}
\hline Tecnología & Principios éticos y valores morales & Fuentes legales y deontológicas \\
\hline $\begin{array}{l}\text { Práctica de la nutrición } \\
\text { clínica }\end{array}$ & $\begin{array}{l}\text { - Atención médica competente } \\
\text { - Compasión y respeto por la dignidad y los derechos humanos } \\
\text { - Principio de autonomía, beneficencia, no maleficencia y justicia } \\
\text { - Dignidad, integridad y vulnerabilidad } \\
\text { - Responsabilidad ética } \\
\text { - Valores morales: atención, responsabilidad, competencia } \\
\text { - Capacidad de respuesta }\end{array}$ & $\begin{array}{l}\text { - Código de ética } \\
\text { - Juramento hipocrático } \\
\text { - Declaración de Cartagena } \\
\text { - Leyes y reglamentos nacionales/ } \\
\text { federales relativos a la nutrición } \\
\text { artificial }\end{array}$ \\
\hline $\begin{array}{l}\text { Innovación en nutrición } \\
\text { clínica y desarrollo de } \\
\text { nutrición administrada } \\
\text { artificialmente }\end{array}$ & $\begin{array}{l}\text { - Responsabilidad } \\
\text { - Principio de autonomía, beneficencia, no maleficencia y justicia } \\
\text { - Principio de explicabilidad } \\
\text { - Relación costo-eficacia, mejor relación beneficios/riesgos- } \\
\text { perjuicios y desarrollo basados en la evidencia } \\
\text { - Respeto del principio de la confidencialidad }\end{array}$ & $\begin{array}{l}\text { - } \text { Declaración de Helsinki } \\
\text { - } \text { Recomendaciones de la UNESCO } \\
\text { sobre la ética en la inteligencia } \\
\text { artificial }\end{array}$ \\
\hline
\end{tabular}

cuando se aplican conjuntamente. Los conceptos de derechos humanos, según Hirsch, caracterizan e iluminan las cuestiones de una exigencia ética en los ámbitos de la asistencia y la investigación ${ }^{(42)}$. En el contexto de los cuidados nutricionales, el respeto de los derechos humanos y la dignidad no son valores abstractos, sino que adquieren una dimensión práctica «que definen un orden social y nos sitúan en obligaciones mutuas entre nosotros» ${ }^{(42)}$. Además, la ética y los derechos humanos son valores que guían a los profesionales de la nutrición clínica, lo que asegura un enfoque centrado en el paciente, donde las necesidades y los derechos de los pacientes son de mayor importancia.

Reconocer el cuidado nutricional como un derecho humano establece un compromiso, con una responsabilidad ética muy importante, que debe basarse en el res- 
peto de los 4 principios éticos (autonomía, beneficencia, no maleficencia y justicia) ${ }^{(43)}$, así como en otros principios, como la vulnerabilidad, la igualdad, la justicia y la equidad $^{(44)}$. Asimismo, siguiendo el concepto de justicia distributiva, los cuidados nutricionales se deben proporcionar de forma equitativa a todos los individuos desnutridos o en riesgo de desnutrición, pero no iguales en el sentido de que, dependiendo de diversos factores, por ejemplo, regionales, culturales, entre otros, estos pueden variar, logrando objetivos similares.

Otro aspecto que engloba los derechos humanos de la justicia distributiva y la T3 se refiere a la prestación de cuidado nutricional cuando los recursos son limitados. Aquí se plantean dos áreas de preocupación: en primer lugar, el concepto de autonomía puede, en algunos casos, considerarse supeditado a las necesidades del estado o de la población en general. Así, el principio del mayor bien para el mayor número prevalece sobre el interés del individuo. En segundo lugar, corresponde a las instituciones de salud desarrollar el marco y los protocolos que rigen la asignación de recursos, mucho antes de que aparezca la escasez ${ }^{(45-48)}$.

Con la esperanzadora aceptación mundial del cuidado nutricional como un derecho humano por parte de gobiernos, legisladores, reguladores y otros interesados, la T3 debería ser menos problemática. Todos los profesionales de la salud deberían conocer la T3 y sus componentes específicos. Estos deben ser embajadores que lleven el mensaje de la T3 y del cuidado nutricional como un derecho humano a los grupos de interés fuera del ámbito de la atención sanitaria. Para ello se necesita tiempo, compromiso y recordar que «primero hay que entender y luego ser entendido» ${ }^{(49)}$.

\section{CONCLUSIÓN}

La tricotomía problemática, definida como el enigma que surge, en gran medida, por la falta de previsión en los aspectos éticos y legales de la aplicación de la tecnología, puede considerarse como un enfoque integral, que debería estar en el centro de la nutrición clínica. Con la esperanzadora aceptación mundial de la atención nutricional como derecho humano, la T3 debería ser menos problemática.

\section{Agradecimientos}

Los autores desean agradecer a los siguientes líderes de opinión sus respectivas contribuciones a la Tabla 1: Bruce Bistrian, Kathy Gura, Dan Waitzberg, Gil Hardy, Sonia Echeverri y Denise Baird Schwartz.

\section{Financiación}

El presente estudio no tuvo financiación.

\section{Conflicto de intereses}

Los autores declaran no tener conflicto de intereses.

\section{Declaración de autoría}

A. Barrocas y D. Cárdenas contribuyeron igualmente a la concepción y diseño del artículo; A. Barrocas contribuyó en la adquisición y análisis de las preguntas a los expertos. A. Barrocas y D. Cárdenas contribuyeron en la interpretación de la información; A. Barrocas y D. Cárdenas redactaron el manuscrito. Todos los autores revisaron el manuscrito, acuerdan ser plenamente responsables de garantizar la integridad y precisión del trabajo, y leyeron y aprobaron el manuscrito final.

\section{Referencias bibliográficas}

1. Barrocas A, Yarbrough G, Becnel PA 3rd, Nelson JE. Ethical and legal issues in nutrition support of the geriatric patient: The can, should, and must of nutrition support. Nutr Clin Pract. 2003;18(1):37-47. doi: 10.1177/011542650301800137

2. Barrocas A. Nutrition support and the troubling trichotomy: A call to action. Nutr Clin Pract. 2006;21(2):109-12. doi: 10.1177/0115426506021002109

3. Barrocas A. The troubling trichotomy 10 years later: Where are we now? Nutr Clin Pract. 2016;31(3):295-304. doi: 10.1177/0884533616629631

4. Cárdenas D, Correia MITD, Ochoa JB, Hardy G, RodríguezVentimilla D, Bermúdez CE, et al. Clinical nutrition and human rights. An international position paper. Nutr Clin Pract. 2021;36(3):534-44. doi: 10.1002/ncp.10667

5. Cárdenas D. What is clinical nutrition? Understanding the epistemological foundations of a new discipline. Clin Nutr ESPEN. 2016;11:e63-6. doi: 10.1016/j.clnesp.2015.10.001

6. Gewirth A. Reason and morality. University of Chicago Press. 1981.

7. Jackson-Meyer K. The principle of proportionality: An ethical approach to resource allocation during the COVID-19 pandemic [Internet]. Bioethics.net. 2020. Consultado el 11 de junio de 2021. Disponible en: https://www.bioethics.net/2020/04/ the-principle-of-proportionality-an-ethical-approach-to-resource-allocation-during-the-covid-19-pandemic/

8. Liddell HG, Scott R. A Greek-English lexicon. $7^{\text {a }}$ edición. New York: Oxford University Press. 1982.

9. Franssen M, Lokhorst GJ, de Poel IV. Philosophy of technology [Internet]. Stanford Encyclopedia of Philosophy. 2018. Consultado el 7 de junio de 2021. Disponible en: https:// plato.stanford.edu/archives/fall2018/entries/technology 
10. Technology [Internet]. Cambridge Dictionary. Consultado el 7 de junio de 2021. Disponible en: https://dictionary.cambridge.org/es-LA/dictionary/english/technology

11. Corbie-Smith G. The continuing legacy of the Tuskegee syphilis study: Considerations for clinical investigation. Am J Med Sci. 1999;317(1):5-8. doi: 10.1097/00000441-19990100000002

12. Brandt AM. Racism and research: The case of the Tuskegee syphilis study. Hastings Cent Rep. 1978;8(6):21-9.

13. Ethical principles and guidelines for the protection of human subjects of research [Internet]. National Commission for the Protection of Human Subjects of Biomedical and Behavioral Research. Consultado el 10 de junio de 2021. Disponible en: https://www.hhs.gov/ohrp/regulations-and-policy/ belmont-report/read-the-belmont-report/index.html

14. Rice TW. The historical, ethical, and legal background of human-subjects research. Respir Care. 2008; 53(10):1325-9.

15. Grodin MA. Historical origins of the Nuremberg code. En: Annas GJ, Grodin MA (editores). The Nazi doctors and the Nuremberg code: Human rights in human experimentation. Oxford: Oxford University Press. 1992. p. 121-44.

16. WMA Declaration of Helsinki-Ethical principles for medical research involving human subjects [Internet]. World Medical Association. 2018. Consultado el 14 de junio de 2021. Disponible en: https://www.wma.net/policies-post/ wma-declaration-of-helsinki-ethical-principles-for-medicalresearch-involving-human-subjects/

17. Cárdenas D, Bermúdez CE, Echeverri S, Pérez A, Puentes M, López L, et al. Declaración de Cartagena. Declaración Internacional sobre el derecho al cuidado nutricional y la lucha contra la malnutrición. Nutr Hosp. 2019;36(4):974-80. doi: $10.20960 /$ nh.02701

18. Cárdenas D, Bermúdez C, Barazzoni R. The Cartagena Declaration: A call for global commitment to fight for the right to nutritional care. Clin Nutr. 2019;38(5):2458-9. doi: 10.1016/j.clnu.2019.07.015

19. Cárdenas D. La nutrition en médecine: approche épistémologique, problèmes éthiques et cas cliniques. Paris: L'Harmattan. 2020.

20. Floridi L, Cowls J. A unified framework of five principles for $\mathrm{AI}$ in society. Harvard Data Science Review. 2019;1(1). doi: $10.1162 / 99608 f 92.8 \mathrm{~cd} 550 \mathrm{~d} 1$

21. Sicard D. L'ethique médicale et la bioéthique. Paris: Presses Universitaires de France. 2009.

22. AMA principles of medical ethics [Internet]. American Medical Association. 2001. Consultado el 1 de abril de 2021. Disponible en: https://www.ama-assn.org/about/publications-newsletters/ama-principles-medical-ethics

23. Potter VR. Bioethics: Bridge to the future. Englewood Cliffs, NJ: Prentice-Hall Pub. 1971.

24. Luppicini R. Technoethics and the Evolving Knowledge Society: Ethical issues in technological design, research, development, and innovation. Estados Unidos: IGI Global. 2010.
25. Heidegger $M$. The question concerning technology and other essays. New York: Harper and Row. 1977.

26. Jonas H. [edición en inglés] The imperative of responsibility: In search of an ethics for the technological age. Chicago/ London: University of Chicago Press. 1984.

27. Maillard N. La vulnérabilité: une nouvelle catégorie morale? Genève: Labor et Fides. 2011.

28. Cárdenas D, Díaz G, Cadavid J, Lipovestky F, Canicoba M, Sánchez $\mathrm{P}$, et al. Nutrition in medical education in Latin America: Results of cross-sectional survey. JPEN J Parenter Enteral Nutr. 2021;1-9. doi: 10.1002/jpen.2107

29. Crowley J, Ball L, Hiddink GJ. Nutrition in medical education: A systematic review. Lancet Planet Health. 2019;3(9):e37989. doi: 10.1016/S2542-5196(19)30171-8

30. Pelluchon C. Eléments pour une éthique de la vulnérabilité. Les hommes, les animaux, la nature. Paris: CERF. 2011.

31. Levinas E. Totalité et infini. Paris: Biblio essais. 1971.

32. Druml C, Ballmer PE, Druml W, Oehmichen F, Shenkin A, Singer $\mathrm{P}$, et al. ESPEN guideline on ethical aspects of artificial nutrition and hydration. Clin Nutr. 2016;35(3):545-56. doi: 10.1016/j.clnu.2016.02.006

33. Tronto J. Un monde vulnérable, pour une politique du care. Paris: Editions La Découverte. 2009.

34. Cederholm T, Barazzoni R, Austin P, Ballmer P, Biolo G, Bischoff SC, et al. ESPEN guidelines on definitions and terminology of clinical nutrition. Clin Nutr. 2017;36(1):49-64. doi: 10.1016/j.clnu.2016.09.004

35. Cederholm T, Jensen GL, Correia MITD, González MC, Fukushima R, Higashiguchi T, et al. GLIM criteria for the diagnosis of malnutrition - A consensus report from the global clinical nutrition community. Clin Nutr. 2019;38(1):1-9. doi: 10.1016/j.clnu.2018.08.002

36. Cárdenas D, Deutz NEP. Is the definition of malnutrition a Sisyphean task? Clin Nutr ESPEN. 2019;29:246-7. doi: 10.1016/j.clnesp.2018.10.005

37. Binet JR. Droit de la bioéthique. Lextenso: Issy les Moulineux. 2017.

38. ASPEN Nutrition Science \& Practice Conference. Poster abstracts. JPEN J Parenter Enteral Nutr. 2021;45:S44S240. Consultado en abril de 2021. doi: 10.1002/jpen.2095

39. Barrocas A, Cohen ML. Have the answers to common legal questions concerning nutrition support changed over the past decade? 10 questions for 10 years. Nutr Clin Pract. 2016;31(3):285-93. doi: 10.1177/0884533616644439

40. Barrocas A, Farquharson J, Fernández M. Legal considerations in nutritional support. Nutr Supp Serv. 1986:6(6):13.

41. Perry JE, Churchill LR, Kirshner HS. The Terri Schiavo case: legal, ethical, and medical perspectives. Ann Intern Med. 2005;143(10):744-8. doi: 10.7326/0003-4819-143-10200511150-00012 
42. Hirsch E, Mann JM. Droits de l'homme et respect de la personne. En: Hirsch E (editor). Traité de bioéthique: fondements, principes repères. Toulouse: Eres. 2010. p. 249-61.

43. Beauchamp TL, Childress FJ. Principles of biomedical ethics. 7a edición. New York: Oxford University Press. 2012.

44. Universal Declaration on Bioethics and Human Rights [Internet]. UNESCO. 2005. Consultado el 1 de abril de 2021. Disponible en: https://en.unesco.org/themes/ethicsscience-and-technology/bioethics-and-human-rights

45. Barrocas A, Schwartz DB, Hasse JM, Seres DS, Mueller CM. Ethical framework for nutrition support resource allocation during shortages: Lessons from COVID-19. Nutr Clin Pract. 2020;35(4):599-605. doi: 10.1002/ncp.10500

46. Berlinger N, Wynia M, Powell T, Hester M, Milliken A, Fabi $\mathrm{R}$, et al. Ethical framework for health care institutions responding to novel Coronavirus SARS-CoV-2 (COVID-19). 2021. Disponible en: https://www.thehastingscenter.org/wp-content/uploads/HastingsCenterCovidFramework2020.pdf
47. Guidelines for institutional ethics services responding to COVID-19, managing uncertainty, safeguarding communities, guiding practice [Internet]. The Hastings Center. 2020. Consultado el 16 de junio de 2021. Disponible en: https:// mk0thehastingsc1jg1a.kinstacdn.com/wp-content/uploads/ HastingsCenterCovidFramework2020.pdf

48. Ethical framework for decision making in HPC during the COVID-19 pandemic [Internet]. National Hospice and Palliative Care Organization. 2020. Consultado el 16 de junio de 2021. Disponible en: https://www.nhpco.org/wpcontent/uploads/COVID-19-Ethical-Framework-DecisionMaking.pdf

49. Habit 5: Seek first to understand then to be understood [Internet]. 10 Minute Leader. 2016. Consultado el 16 de junio de 2021]. Disponible en: https://www.the10minuteleader. com/habit-5-seek-first-to-understand-then-to-be-understood/ 